\title{
LAMELLOCYTE DIFFERENTIATION IN Drosophila LARVAE PARASITIZED BY Leptopilina
}

\author{
T. M. Rizki and R. M. Rizki \\ Department of Biology, University of Michigan, Ann Arbor, MI 48109 \\ (Submitted July 1990; Accepted July 1991)
}

\begin{abstract}
$\square$ Abstract-The presence of Leptopilina heterotoma or Leptopilina boulardi eggs in the hemocoel of a Drosophila melanogaster larva induces the differentiation of lamellocytes, the blood cells that encapsulate foreign objects. $L$. boulardi eggs are encapsulated by the newly differentiated lamellocytes, but $L$. heterotoma eggs are not. The induced lamellocytes in host larvae with $L$. heterotoma eggs undergo the same destructive morphological changes as reported previously for lamellocytes present in melanotic tumor mutant larvae at the time of parasitization. Thus, the virus-like particles produced by the $L$. heterotoma female to protect its eggs from encapsulation do not block the differentiation of lamellocytes, but rather destroy lamellocytes whenever they are present in the hemocoel.
\end{abstract}

$\square$ Keywords-Parasitoid; Drosophila; Blood cells; Encapsulation; Lamellocyte.

\section{Introduction}

Cellular capsules that form around foreign objects and abnormal tissues recognized as not-self in the hemocoel of melanotic tumor larvae of Drosophila melanogaster consist of layers of lamellocytes $(1,2)$. Lamellocytes are discoidal blood cells with sticky surfaces $(3,4)$. In melanotic tumor larvae parasitized by Leptopilina heterotoma, a cynipid wasp, the lamellocytes lose their surface adhesiveness and become bipolar cells (5). These modified lamellocytes are incapa-

Address correspondence to T. M. Rizki, Department of Biology, University of Michigan, Ann Arbor, MI 48109. ble of forming capsules and are eventually destroyed so the parasitoid eggs in the host hemocoel develop unmolested by the type of host blood cell potentially harmful to them. It has been demonstrated recently that the destructive factor for host lamellocytes is a virus-like particle from an accessory gland of the female wasp's reproductive system (6). No detrimental effects on the plasmatocytes and crystal cells of parasitized hosts have been observed (5), suggesting that the virus-like particles injected into the host along with eggs by the parasitoid female selectively destroy host lamellocytes.

To explain the low frequency of lamellocytes in hosts parasitized by $L$. heterotoma, earlier workers concluded that lamellocyte differentiation is suppressed in parasitized hosts $(7-10)$. Recent in vitro studies confirmed that lamellocytes are destroyed by $L$. heterotoma viruslike particles (11) so the decreased frequency of lamellocytes in parasitized hosts can be explained by the selective destruction of the cells. Whether lamellocyte differentiation is also suppressed in parasitized hosts is not excluded by the demonstration that the virus-like particles destroy lamellocytes. Therefore, the present study was undertaken to examine the earlier suggestion $(7-10)$, which, if tenable, would support the contention that the virus-like particles play more than one role in curbing lamellocyte activity.

Melanotic tumor larvae have many lamellocytes that are competent to en- 
capsulate aberrant tissues developing under the influence of mutant genes (12). Therefore, melanotic tumor larvae are ideal hosts to examine the effects of parasitization on a cellular defense system already primed to combat foreign materials. The presence of lamellocytes in hosts at the time of parasitoid oviposition complicates the investigation of lamellocyte differentiation as a response to parasitization. Ideal hosts to examine this question should lack lamellocytes initially, yet be capable of producing these cells. The frequency of lamellocytes is low in wild-type $D$. melanogaster larvae whose cellular defense system has not been challenged by the presence of a foreign object (3). The present study used larvae from an inbred strain of wild-type $D$. melanogaster that have no or few lamellocytes during most of the third instar. To assure that larvae of this strain can respond to parasitization by producing lamellocytes and encapsulating parasitoid eggs, we used $L$. boulardi wasps routinely encapsulated in $D$. melanogaster hosts (13-15) as controls.

A parasitoid egg is a foreign object in the hemocoel of its host, so we might presume that lamellocyte differentiation in the parasitized larva is induced by the presence of these foreign objects in the hemocoel. An additional consequence of parasitization is injury imposed by the female wasp's ovipositor piercing the host cuticle to inject its egg. The site of this small puncture heals and remains visible as a minute, dark spot. When Drosophila larvae are injected with foreign materials, the injured cuticle heals to leave a black crust at the injection site (16). Since it has been demonstrated that injury induces lamellocytes and melanotic masses in Drosophila larvae (17), the trauma associated with parasitoid oviposition might stimulate lamellocyte differentiation. To partition the effect of the parasitoid egg from the injury due to insertion of the wasp ovipositor, we deter- mined lamellocyte frequencies in injured Drosophila larvae and in larvae injected with foreign objects.

\section{Materials and Methods}

\section{Parasitoid and Host Strains}

The Leiden strain of $L$. heterotoma was bred on a temperature-sensitive, melanotic tumor stock of $D$. melanogaster, $t u-S z^{t s}$, and the L104 strain of $L$. boulardi was bred on the Brazzaville strain of $D$. melanogaster at $25^{\circ} \mathrm{C}$ (14). Adult wasps were maintained at $18^{\circ} \mathrm{C}$ on $50 \%$ honey $/ \mathrm{H}_{2} \mathrm{O}$ solution. Two- to 4-week-old females that had not been exposed to Drosophila larvae previously were used for the experiments.

Hosts were from a stock of wild-type D. melanogaster designated Ore- $R$ line B5. This line was set up as a single-pair mating of flies from an Ore- $\mathrm{R}-\mathrm{CH}$ stock in 1976 and the line has been kept in mass culture since that time. Larvae emerging from eggs within a 2 -h period were transferred to fresh-food containers. Ages of larvae were recorded as \pm 1 $h$ from egg eclosion. The larvae were grown at $25-27^{\circ} \mathrm{C}$ on Drosophila cream of wheat/molasses medium seeded with live Fleischmann's yeast.

\section{Parasitization Experiments}

Drosophila larvae were rinsed in distilled $\mathrm{H}_{2} \mathrm{O}$ when they were $48 \mathrm{~h}$ old (early third instar) and transferred to filter paper moistened with $0.2 \%$ glucose solution. The filter paper with larvae was placed in a plastic vial containing adult wasps. Two hours later the wasps were removed and the Drosophila larvae were returned to regular Drosophila medium. One vial with unparasitized Drosophila 
larvae on glucose-moistened paper was maintained as the control group. These larvae were returned to containers with regular medium after $2 \mathrm{~h}$.

Two experiments were run using nine batches of Drosophila larvae. The same ages of Drosophila larvae and wasps were used for the nine groups so data could be pooled for statistical analysis.

\section{Drosophila Blood Cell Samples}

To determine lamellocyte frequency at the beginning of each experiment, hemolymph samples were taken from 48 - $h$ old larvae. The remaining larvae in the batch were divided into two groups, one exposed to wasps and the other maintained as the control (unparasitized). The parasitized and unparasitized larvae were bled 4,24 , and $48 \mathrm{~h}$ after they had been returned to regular medium. Most of the larvae examined $48 \mathrm{~h}$ after parasitization had left the culture medium and were on the walls of the containers preparing to pupariate. Parasitoid larvae had hatched in these hosts.

Hemolymph from individual larvae was collected in a drop of phosphatebuffered saline for Drosophila cells (18). After removing the hemolymph sample from a larva that had been exposed to parasitoids, the larva was transferred to another saline drop and dissected to search for parasitoid eggs to confirm that it had been parasitized. The blood cells in the hemolymph samples were examined with phase optics and all cells in each sample were counted. Lamellocytes in the samples were scored as discoidal (normal) or elongated (affected).

\section{Injury Experiments}

Experiments to evaluate the effects of injury on lamellocyte differentiation used 48-h-old Drosophila larvae that had been etherized to immobilize them during the operation. Some larvae were injected with glass beads (mesh size 200400) in phosphate-buffered saline. The same needle was used to inject a second group of larvae with saline alone. A third group of larvae was punctured with a metal needle that caused a cuticle wound about the size of that in the first two groups of larvae. Larvae of a fourth group were pricked with a metal microneedle made by electropolishing a $0.020^{\prime \prime}$ diameter tungsten wire to a point in $2 \%$ $\mathrm{NaOH}$ solution (19). The tips of these needles are so fine that they were used previously for microdissection of crystal cells while the cells were viewed in a scanning electron microscope (20). This size of needle tip was selected since it approximates the size of the Leptopilina ovipositor. Hemolymph samples were taken from the four groups of larvae $24 \mathrm{~h}$ after they had been injected or injured. The blood cells were examined and counted as in the previous experiments.

\section{Statistical Analysis}

The percent lamellocytes (normal + affected) was calculated for each hemolymph sample (= each larva). Examination of the ranges of lamellocyte percentages for the larvae given the same treatment in both experiments with parasitoids showed that the values overlapped. For example, the percentage values for the hosts sampled $24 \mathrm{~h}$ after parasitization by $L$. heterotoma in the first experiment were within the range for the values from this same treatment in the second experiment. Therefore, the data from both experiments were pooled for statistical analysis.

The lamellocyte percentage for each blood cell sample was converted into an- 
gle $=\arcsin \sqrt{\text { percentage }}(21)$ as used previously for samples of Drosophila blood cells (3). In cases of $0-5 \%$ or $95-$ $100 \%$, an angular value of 2.87 corresponding to $0.25 \%$ was added or subtracted, respectively. The mean angle for each group of larvae was then calculated and the angle values for the means were used to evaluate treatments by Student's $t$ statistics (21). A significance level of $p$ $\leqslant 0.05$ was selected for sample comparisons.

\section{Results}

\section{Lamellocytes in Unparasitized Drosophila Larvae}

Data on the blood cells of the control groups of Drosophila larvae are summarized in Table 1. No lamellocytes were found in the hemolymph samples taken from 48-h-old larvae (control, $0 \mathrm{~h}$ ), the age at which all other larvae were exposed to parasitoid females or transferred to glucose solution for the control series. Lamellocyte frequency remained low in the control groups during the subsequent $48 \mathrm{~h}$ of observation (Table 1).

\section{Lamellocytes in Parasitized Drosophila Larvae}

None of the larvae sampled (total of 19) $4 \mathrm{~h}$ after parasitization by $L$. heterotoma or $L$. boulardi had lamellocytes (Table 1). Twenty-four hours after parasitization, all hemolymph samples from larvae with parasitoid eggs had lamellocytes. Only 1 lamellocyte was found in the total of 2363 cells from 9 control larvae at this age so the lamellocyte increase in both groups of parasitized larvae is significant, as indicated in Table 1. The frequency of lamellocytes in hosts with $L$. boulardi eggs was higher than in hosts with $L$. heterotoma eggs (Fig. 1). As reported in earlier studies $(5,14,15)$, no lamellocytes were found adhering to $L$. heterotoma eggs, but $L$. boulardi eggs were encapsulated by lamellocytes. The lamellocytes in hosts with $L$. boulardi eggs were normal in shape, but elongated lamellocytes as well as the normal, discoidal lamellocytes were present in hemolymph samples from hosts with $L$. heterotoma eggs (Table 1, Fig. 2).

Table 1. Lamellocytes in parasitized and unparasitized Drosophlla larvae.

\begin{tabular}{|c|c|c|c|c|c|}
\hline \multirow[b]{3}{*}{ Sample } & \multirow{3}{*}{$\begin{array}{l}\text { Number } \\
\text { of Larvae }\end{array}$} & \multicolumn{3}{|c|}{ Number of Blood Cells } & \multirow{3}{*}{$\begin{array}{c}\text { Angles Corresponding } \\
\text { to Lamellocyte } \\
\text { Percentages } \\
(\bar{S} \pm \mathrm{SE})\end{array}$} \\
\hline & & \multirow[b]{2}{*}{ Total } & \multicolumn{2}{|c|}{ Lamellocytes } & \\
\hline & & & Normal & Affected & \\
\hline \multicolumn{6}{|l|}{ Control } \\
\hline $\mathrm{Oh}$ & 6 & 1945 & 0 & 0 & $2.87-$ \\
\hline $4 \mathrm{~h}$ & 10 & 1702 & 1 & 0 & $3.35 \pm 0.48$ \\
\hline $24 \mathrm{~h}$ & 9 & 2363 & 1 & 0 & $3.21 \pm 0.34$ \\
\hline $48 \mathrm{~h}$ & 9 & 7159 & 6 & 0 & $3.45 \pm 0.58$ \\
\hline \multicolumn{6}{|l|}{$\begin{array}{l}\text { Parasitized } \\
\text { L. heterotom }\end{array}$} \\
\hline $4 h$ & 10 & 5564 & 0 & 0 & 2.87 \\
\hline $24 h$ & 10 & 7831 & 466 & 298 & $18.18^{*} \pm 1.58$ \\
\hline $48 h$ & 9 & 6674 & 21 & 45 & $6.74 \pm 1.30$ \\
\hline \multicolumn{6}{|l|}{ L. boulardi } \\
\hline $4 h$ & 9 & 5060 & 0 & 0 & 2.87 \\
\hline $24 h$ & 9 & 2849 & 1112 & 0 & $41.50^{*} \pm 3.60$ \\
\hline $48 h$ & 10 & 6908 & 2506 & 0 & $38.21^{\star} \pm 3.59$ \\
\hline
\end{tabular}

* Differs significantly from the control at the same hour. 


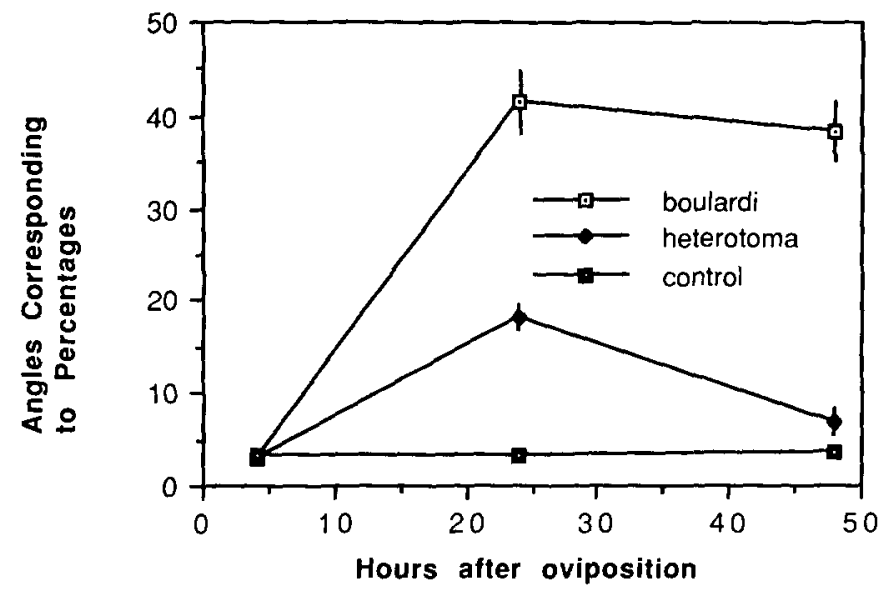

Figure 1. Changes in host lamellocyte frequencies as a function of time after parasitoid oviposition.

Lamellocyte frequency was lower in hosts that had been parasitized for $48 \mathrm{~h}$ than in hosts sampled at $24 \mathrm{~h}$ postoviposition. The decrease in lamellocytes in the hosts parasitized by $L$. heterotoma was noticeably greater than the lamellocyte decrease in the hosts with $L$. boulardi eggs and larvae (Fig. 2). The difference between the means obtained for the 24- and 48-h samples of $L$. boulardi is not statistically significant $\left(t_{\mathrm{df} 17}=0.65 ; p>\right.$ $0.50)$, whereas the difference between the means of the $L$. heterotoma 24- and 48-h samples is significant $\left(t_{\mathrm{df} 17}=2.69\right.$; $p<0.025$ ). The $L$. heterotoma sample taken at $48 \mathrm{~h}$ did not differ significantly from the control sample at $48 \mathrm{~h}$, whereas the $L$. boulardi sample at $48 \mathrm{~h}$ did (Table 1). As the means obtained for the 24- and 48-h $L$. boulardi samples did not differ significantly, the values for these samples were pooled and the pooled sample compared with the $L$. heterotoma 24-h sample. The difference between the means of these groups is statistically significant $\left(t_{\mathrm{df} 27}=5.63 ; p<0.001\right)$.

Both normal and affected lamellocytes were present in hosts that had been parasitized for $48 \mathrm{~h}$ by $L$. heterotoma, but the lamellocytes in hosts with $L$. boulardi eggs and larvae were normal in shape (Table 1).

\section{Effects of Injury}

The data from the injury and injection experiments with Drosophila larvae are summarized in Table 2. Hemolymph samples from larvae injected with glass beads had lamellocytes when they were examined $24 \mathrm{~h}$ postinjection. Small melanotic masses consisting of glass beads surrounded by lamellocytes were present in the larvae. The larvae also had small melanotic masses that did not contain glass beads but did have lamellocytes. Cellular masses surrounded by lamellocytes were also found in larvae injected with buffer alone, and hemolymph samples from these larvae contained lamellocytes. The frequency of lamellocytes in larvae injected with buffer was not significantly different from that of larvae injected with glass beads $\left(t_{\mathrm{df} 6}=1.53 ; p>0.10\right)$.

Larvae receiving puncture wounds from a large metal needle had lamellocytes and small melanotic masses similar to those seen in larvae injected with buffer solution. Lamellocyte frequency in these larvae did not differ significantly from lamellocyte frequency in larvae injected with buffer solution $\left(t_{\mathrm{df} 8}=0.35\right.$; $p<0.50)$ or with glass beads $\left(t_{\mathrm{df} 8}=\right.$ $1.83 ; p>0.10$ ). No lamellocytes or mel- 

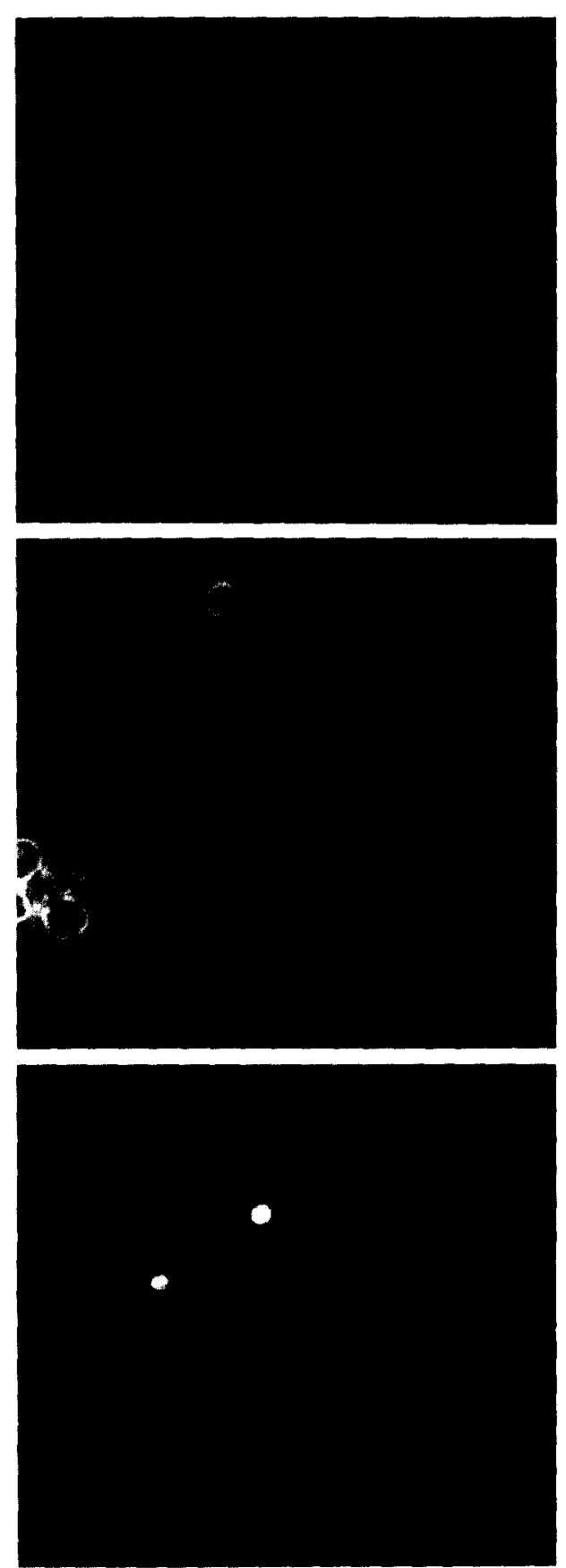

Figure 2. Drosophila lamellocytes: (a), from unparasitized larvae; (b), from $L$. boulardi parasitized larvae; (c), from larvae parasitized by $L$. heterotoma. The elongated lamellocytes in (c) are from two different fields of the same hemocyte sample. The small round cells with the bright phase contrast are plasmatocytes. Scale bar $=$ $25 \mu \mathrm{m}$. anotic masses were found in larvae that had been punctured with the microneedle. The puncture sites had healed and were visible as minute, dark spots.

\section{Discussion}

Parasitization by $L$. boulardi or $L$. heterotoma induces lamellocytes in the hemocoel of Drosophila larvae. The lamellocytes appear within $24 \mathrm{~h}$ of parasitization by both parasitoid species. However, the fate of the newly differentiated lamellocytes differs in the hosts with $L$. heterotoma and $L$. boulardi eggs. Newly differentiated lamellocytes encapsulate $L$. boulardi eggs, but the induced lamellocytes in the hosts with $L$. heterotoma eggs are incapacitated and destroyed in the same manner as reported previously for lamellocytes present in melanotic tumor larvae at the time of parasitization $(5,14)$. Thus, the virus-like particles of $L$. heterotoma females function to destroy lamellocytes produced in the host, not to block the differentiation of lamellocytes.

In hosts with $L$. boulardi eggs, lamellocyte frequency did not change significantly between 24 and 48 h postoviposition. It should be noted, however, that the percentage of lamellocytes calculated for hemolymph samples taken from these hosts does not represent the true frequency of this type of blood cell in the larva since lamellocytes that encapsulate the $L$. boulardi eggs are removed from circulation and thus are excluded from the sampling $(14,15)$. Lamellocytes are not utilized for cellular capsules in hosts with $L$. heterotoma eggs. These hosts showed a significant decrease in lamellocyte frequency between 24 and $48 \mathrm{~h}$ postoviposition. As some of the lamellocytes in these hosts showed the harmful effects caused by $L$. heterotoma viruslike particles (5), the decline in lamello- 
Table 2. Lamellocytes in injured Drosophlla larvae.

\begin{tabular}{|c|c|c|c|c|}
\hline \multirow[b]{2}{*}{ Treatment } & \multirow{2}{*}{$\begin{array}{l}\text { Number } \\
\text { of Larvae }\end{array}$} & \multicolumn{2}{|c|}{ Number of Blood Cells } & \multirow{2}{*}{$\begin{array}{l}\text { Angles Corresponding } \\
\text { to Lamellocyte } \\
\text { Percentages } \\
(X \pm S E)\end{array}$} \\
\hline & & Total & Lamellocytes & \\
\hline \multicolumn{5}{|l|}{ Injection } \\
\hline Glass beads & $4^{*}$ & 1492 & 334 & $31.79 \pm 4.21$ \\
\hline Buffer & $4^{\star}$ & 1535 & 178 & $20.67 \pm 2.94$ \\
\hline \multicolumn{5}{|l|}{ Puncture wound } \\
\hline Large & $6^{*}$ & 5736 & 556 & $18.51 \pm 3.06$ \\
\hline Small & 5 & 4398 & 0 & $2.87-$ \\
\hline
\end{tabular}

* Melanotic masses in the hemocoel.

cyte frequency in the hosts must result from destruction of the cells.

Glass beads injected into the hemocoel of a Drosophila larva induced lamellocytes that encapsulated the beads to form small melanotic masses. The melanotic masses that did not contain beads in these larvae probably resulted from encapsulation of tissues injured during the operations. The latter suggestion is supported by the observations that similar small masses were found in larvae injected with buffer solution and in larvae wounded without injecting foreign materials. Earlier studies showed that internal injuries resulting from mechanical handling of Drosophila larvae cause melanotic masses to form around injured tissues (17). Implanted Drosophila tissues devoid of basement membrane are encapsulated by lamellocytes, supporting the suggestion that the basement membrane covering all surfaces in the insect hemocoel is recognized as self by the blood cells and the absence of this covering denotes not self to the blood cells $(22-24)$. Injection of foreign materials and infliction of wounds with rela- tively large-bore needles must damage basement membrane or dislodge cells and tissue fragments so they are not protected by basement membrane. These tissues with damaged basement membrane represent not self so lamellocyte differentiation is induced. The newly differentiated lamellocytes encapsulate the damaged tissues to form melanotic masses.

The minute punctures made in the cuticles of the Drosophila larvae with a microneedle did not induce lamellocytes or cause melanotic masses. The punctures healed to form minute, dark specks similar to those found in the cuticles of parasitized larvae. Therefore, it is unlikely that the slight injury to the host body wall inflicted by the female wasp ovipositor is responsible for lamellocyte differentiation in parasitized Drosophila larvae. The presence of the foreign objects, i.e., parasitoid eggs, must induce lamellocyte differentiation.

Acknowledgement-This research was supported by NIH Grant GM-37025.

\section{References}

1. Rizki, R. M.; Rizki, T. M. Cell interactions in the differentiation of a melanotic tumor in Drosophila. Differentiation 12:167-178; 1979.

2. Rizki, T. M.; Rizki, R. M. Surface changes on hemocytes during encapsulation in Drosophila melanogaster Meigen. In: Gupta, A. P., ed.
Hemocytic and humoral immunity in arthropods. New York: John Wiley \& Sons, Inc.; 1986:157-190.

3. Rizki, T. M. Alterations in the hemocyte population of Drosophila melanogaster. J. Morphol. 100:437-458; 1957. 
4. Rizki, T. M.; Rizki, R. M. Blood cell surface changes in Drosophila mutants with melanotic tumors. Science 220:73-75; 1983.

5. Rizki, R. M.; Rizki, T. M. Selective destruction of a host blood cell type by a parasitoid wasp. Proc. Natl. Acad. Sci. USA 81:6154$6158 ; 1984$.

6. Rizki, R. M.; Rizki, T. M. Parasitoid virus-like particles destroy Drosophila cellular immunity. Proc. Natl. Acad. Sci. USA 87:8388$8392 ; 1990$.

7. Walker, 1. Die abwehrreaktion des wirtes Drosophila melanogaster gegen die zoophage cynipide Pseudeucoila bochei Weld. Rev. Suisse Zool. 66:569-632; 1959.

8. Nappi, A. J. Cellular immune reactions of larvae of Drosophila algonquin. Parasitology 70:189-194; 1975.

9. Nappi, A. J.; Streams, F. A. Haemocytic reactions of Drosophila melanogaster to the parasites $P$ seudocoila mellipes and $P$. bochei. J. Insect Physiol. 15:1551-1566; 1969.

10. Nappi, A. J.; Silvers, M. Cell surface changes associated with cellular immune reactions in Drosophila. Science 225:1166-1168; 1984.

11. Rizki, R. M.; Rizki, T. M. Effects of lamellolysin from a parasitoid wasp on Drosophila blood cells in vitro. J. Exp. Zool. 257:236-244; 1991.

12. Rizki, T. M. Tumor formation in relation to metamorphosis in Drosophila melanogaster. $\mathrm{J}$. Morphol. 100:459-472; 1957.

13. Carton, Y.; Bouletreau, M.; van Alphen, J. J. M.; van Lenteren, J. C. The Drosophila parasitic wasps. In: Ashburner, M.; Thompson, J., eds. The genetics and biology of Drosophila, vol. 3e. London: Academic Press. 1986:347-394.

14. Rizki, T. M.; Rizki, R. M.; Carton, Y. Strategies to avoid cellular defense responses of
Drosophila melanogaster. Exp. Parasitol. 70:466-475; 1990.

15. Rizki, R. M.; Rizki, T. M. Encapsulation of parasitoid eggs in phenoloxidase-deficient mutants of Drosophila melanogaster. J. Insect Physiol. 36:523-529; 1990.

16. Rizki, T. M.; Rizki, R. M. The cellular defense system of Drosophila melanogaster. In: King R. C.; Akai, H., eds. Insect ultrastructure, vol. 2. New York: Plenum Press; 1984:579_ 604.

17. Rizki, T. M. Melanotic tumor formation in Drosophila. J. Morphol. 106:147-158; 1960.

18. Seecof, R. L. Phosphate-buffered saline for Drosophila. Drosophila Inform. Serv. 46:113; 1971.

19. Rizki, T. M.; Allard, L. F.; Rizki, R. M.; Bigelow, W. C. Scanning electron microscopy and microdissection of single Drosophila cells. Annu. Proc. Electron Microscopy Soc. Am. 33:610-611; 1975

20. Rizki, T. M.; Rizki, R. M.; Allard, L. F.; Bigelow, W. C. Micromanipulation of tissues and cells of the Drosophila larva in the SEM. Scan. Electron Microscopy 8:611-618; 1976.

21. Snedecor, G. W.; Cochran, W. G. Statistical methods. Ames, IA: The Iowa State University Press; 1980.

22. Salt, G, The cellular defense reactions of insects. Cambridge, UK: Cambridge University Press; 1970.

23. Rizki, R. M.; Rizki, T. M. Hemocyte responses to implanted tissues in Drosophila melanogaster larvae. Roux's Archiv. Dev. Biol. 189:207-213; 1980.

24. Rizki, R. M.; Rizki, T. M.; Bebbington, C. R.; Roberts, D. B. Drosophila larval fat body surfaces: changes in transplant compatibility during development. Roux's Archiv. Dev. Biol. 192:1-7; 1983. 